Products and Services In Practice is provided to readers using text and images from the manufacturer, supplier or distributor and does not imply endorsement by $B D J$ In Practice. Normal and prudent research should be exercised before purchase or use of any product mentioned.

Please send product and services news through to David Westgarth, $B D J$ In Practice via: David.Westgarth@bda.org

\section{Safe Airflow education in a brave new world}

EMS and the Swiss Dental Academy (SDA) are delighted to announce that their ever-popular Guided Biofilm Therapy (GBT) events are back on track, with new protocols to ensure everyone's safety.

The courses are ideal for dental hygienists and therapists looking to offer their patients the very best in prophylaxis and to renew their vigour for dentistry in these challenging times, while achieving a financial benefit.

Currently, the SDA is offering the GBT Masterclass, a half-day of theoretical knowledge to develop strategies for periodontal and implant maintenance by looking at the components of the GBT model including AirFlow, PerioFlow and the advancements in 'No Pain' Piezon technology.

The GBT protocol is based on individual patient diagnosis and risk assessment in order to achieve optimal results. The treatment is given in the least invasive way, with the highest level of comfort, safety and efficiency. This means that even previously anxious patients are happy to return time and again, boosting practice income on a regular basis.

Course numbers are limited to 10 , in a space suitable to hold at least 20 delegates, so that social distancing can be easily achieved. The organiser will also be printing QR codes for track and trace, keeping windows and doors open, plus providing hand sanitiser on arrival and masks to whomever wants them.

For further information and to book, visit https://bit.ly/30lKZPh.

\title{
Support for mouth cancer patients
}

Many mouth cancer-related deaths can be prevented if the disease is identified early and treated quickly enough.

If you suspect a possible mouth cancer case, or have a patient recovering from cancer treatment, you can always refer to Ten Dental+Facial for a second opinion. Equally, the multi award-winning team can provide professional support in managing the patient's oral condition.

Ten Dental+Facial has streamlined its internal procedures to make referring as seamless and hassle-free as possible. You will remain integral to the process - benefitting from regular updates as treatment progresses - before the patient is returned to your care.
With Ten Dental+Facial as your trusted referral partner, you can ensure mouth cancer patients are optimally supported.

For more information visit www. tendental.com or call on 02033932623.

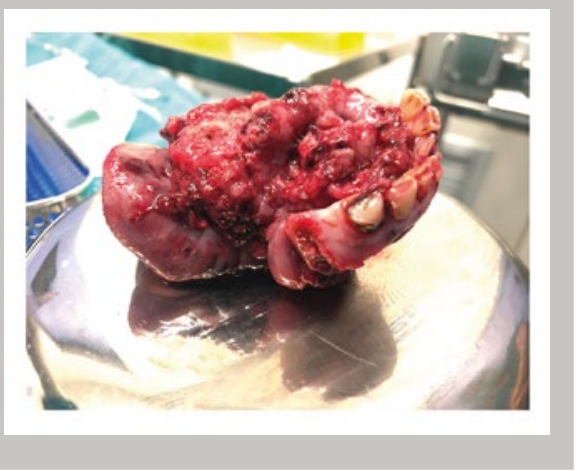

\section{Sensitivity relief}

Everyone doesn't welcome crisp cold mornings; those suffering from dental sensitivity can find this time of year challenging to say the least. Experienced by a wide cross-section of the population, most notably when eating or drinking cold or sweet food, or taking a gasp of cold air, sensitivity is caused by nerves which are stimulated in the dentine layer of teeth exposed by, amongst other things, tooth wear or periodontal disease.

Sensitivity is often experienced in conjunction with gum irritation. Oral-B know that strong teeth are built on healthy gums. This was the premise behind the launch of their new toothpaste, Oral-B Sensitivity \& Gum Calm. It contains

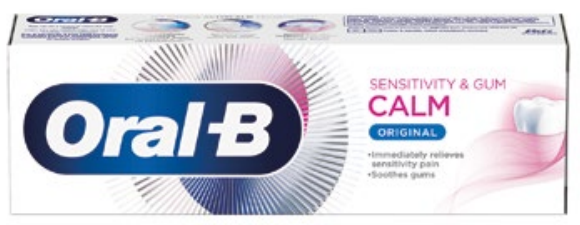

stabilised stannous fluoride, which acts by blocking the dentinal tubules and thus preventing the stimuli causing the painful nerve impulses. Used regularly it will continue to block the tubules thereby providing ongoing sensitivity relief.

The inclusion of stabilised stannous fluoride also gives Oral-B's Sensitivity \& Gum Calm toothpaste an antimicrobial action, which fights plaque and consequently gum problems. The stabilised stannous fluoride will inhibit antimicrobial growth as well as reducing the ability of bacteria to 'stick' to tooth and gum surfaces.

All toothpastes are not the same. Whilst the inclusion of fluoride is a taken, to afford maximum protection, a formulation must contain other proven ingredients that work in harmony together to promote good oral health. Oral-B Sensitivity \& Gum Calm is formulated to strengthen teeth and soothe what they are built on, gums! 